\title{
Opinión pública en Chile durante la unidad popular: Una revisión de "la tesis de la polarización"
}

\author{
Public Opinion in Chile during the period of the Popular Unity: \\ A review of "The Polarization Thesis"
}

Lucía Miranda Leibe* - Renata Retamal Iturriaga**

\begin{abstract}
Resumen: En la historia política de Chile el proceso que conduce al golpe de Estado en 1973 se explica a través de la amplia asunción de la polarización política de la sociedad que requería "recurrir a los militares como garantes de la democracia" 1 . En este trabajo a partir de la distinción entre polarización y politización se busca responder ¿en qué medida la polarización ideológica manifestada a nivel de la élite durante 1970, ilustra el posicionamiento político-ideológico de las masas en Chile? es decir, para el periodo previo al golpe de Estado en Chile ¿estaban los ciudadanos polarizados ideológicamente como lo estaban las élites partidarias? Estas preguntas son respondidas a partir de los datos de encuestas que periódicamente llevó a cabo el sociólogo Eduardo Hamuy durante 1970 y 1973. Los datos se analizan desde la llegada a la presidencia de Salvador Allende en 1970 hasta los meses previos a su derrocamiento. Finalmente, se concluye que, si bien la politización era alta, no existía polarización a nivel de la ciudadanía chilena. A su vez, se constata un predominante rechazo a la pertinencia de un gobierno militar para Chile.
\end{abstract}

Palabras claves: polarización, politización, rechazo a un gobierno militar, Chile, encuestas Hamuy.

\begin{abstract}
In the political history of Chile, the process leading to the coup d'état in 1973 is explained by the widespread assumption of the political polarization of society that required "recourse to the military as guarantors of democracy." In this work, based on the distinction between polarization and politicization, we seek to answer to what extent does the ideological polarization manifested at the elite level during 1970 illustrate the political-ideological positioning of the masses in Chile? that is, for the period prior to the coup d'état in Chile, were the citizens polarized ideologically as were the party elites? These questions are answered from the survey data periodically carried out by the sociologist Eduardo Hamuy during 1970 and 1973. The data are analyzed from the arrival to the presidency of Salvador Allende in 1970 until the months before his overthrow. Finally, it is concluded that although politicization was high, there
\end{abstract}

*(FLACSO-Chile), Dra. Estudios Internacionales lucia.miranda@flacsochile.org nacionalidad argentina-española autora principal

**(FLACSO-Chile), Cientista Política renata.retamal@flacsochile.org rvretama@uc.cl nacionalidad chilena autora secundaria

1. Isabel Torres. "La Crisis del Sistema Democrático: las elecciones presidenciales y los proyectos políticos excluyentes. Chile 1958-1970", Santiago, Editorial Universitaria, 2014 (pág.17). 
was no polarization at the level of Chilean citizenship. At the same time, there is a predominant rejection of the relevance of a military government for Chile.

Keywords: polarization, politicization, rejection of a military government, Chile, Hamuy polls.

Recibido: 2 julio 2018 Aceptado: 28 agosto 2018

\section{Introducción}

El período previo al golpe de Estado de 1973 es descrito por los libros de historia en Chile como un momento de fuerte crisis al borde de una guerra civil. El discurso en torno al alto nivel de tensión entre las posturas políticas opuestas llamaba y justificaba "recurrir a los militares como garantes de la democracia" 2 . Desde este paradigma se desprende el concepto de "polarización", que es entendida de manera general como un factor que "contribuye al quiebre de la democracia, corrupción y declive económico"3. El objetivo de este artículo consiste en demostrar que tal nivel de polarización política transcurría sólo a nivel de las élites políticas y no a nivel de la ciudadanía.

En este artículo se considera élite política a quienes detentan poder político; es decir, los políticos, organizaciones políticas, incluida la prensa. La prensa chilena ha tendido a ser calificada como homogénea en términos de contenido ideológico coincidiendo la propiedad por parte de los dueños de los principales periódicos con la ostentación del poder económico ${ }^{4}$. Asimismo, es importante destacar que para la época los periódicos estaban claramente alineados a los grupos políticos ${ }^{5}$ (Faure 2016: 3): El Siglo al partido comunista (PC) como periódico partidista; Clarín vinculado a Víctor Pey (cercano de Allende) y, El Mercurio y La Tercera como los dos periódicos vinculados a las grandes familias conservadoras de la época (los Edwards y los Picó Cañas). Si bien se reconoce que existe una amplia literatura que por un lado rechaza la contribución de la prensa al golpe de Estado6 6 , acá dicho debate queda fuera de contemplación ya que al

2.Idem.

3. Entre quienes sostienen esta tesis se encuentran Juan Linz. "The Breakdown of Democratic Regimes: Crisis, Breakdown And Reequilibration”, Baltimore, Johns Hopkins University Press, 1978 (pág.44). También se sugiere revisar Noam Lupu. "Party Polarization and Mass Partisanship: A Comparative Perspective", Polital Behavior, Vol.37, No.2, Kentucky, 2014, 331-356 y, adicionalmente, Arturo Valenzuela. "Democracy, Politics, and Society Latin America: Essays in Honor of Juan Linz", Boulder, Westview Press, 2003.

4.Gronemeyer, María Elena y Porath, William. "Tendencias de la posición editorial en diarios de referencia en Chile. El arte de dosificar la crítica frente a la actuación de los actores políticos", Revista de Ciencia Política, Vol. 37, No.1, Santiago, 2017, (pág. 181).

5.Antoine Faure. "Par tempsagités Temporalités journalistiques et crise politique au Chili (1970-1990)", Temporalite, Vol.23, 2016, (pág. 3).

6. Para más información ver en Patricio Bernedo y William Porath. "A tres décadas del golpe: ¿Cómo contribuyó la prensa al quiebre de la democracia chilena?" Cuadernos de Información. No.16-17, Santiago, 2003-2004, 114124 y también Faure, 2016, op. cit. 
entenderse que la prensa pertenecía a la élite política se excluye del nivel de observación en que se pone interés: la ciudadanía ${ }^{7}$.

En este trabajo, por medio de la descripción del contexto e interpretación del ambiente -a partir de los resultados de simpatía partidaria y autoubicación ideológica presentada por las encuestas Hamuy ${ }^{8}$ se revisa y refuta la tesis de la polarización política en la ciudadanía previa a la intervención militar de 1973; a la vez que se reconoce una fuerte politización. El abordaje de las conclusiones de este trabajo a partir de la provisión de datos empíricos a nivel de ciudadanía es uno de los principales aportes de este estudio, que permiten refutar precisamente con datos de encuesta de la época la tesis de la polarización ciudadana previa al golpe. En este sentido se entiende que el colapso democrático se debió a la polarización de la élite (líderes en el ejército, gobierno y grupos de interés), y no porque los votantes abandonaran los partidos centristas ${ }^{9}$.

El estudio de la historia política a partir de la identificación e interpretación de ciertos hechos por sobre otros, tiene un contenido ideológico provocando que ciertos pasados se impongan al presente; en este sentido, se entiende que los eventos históricos como objeto de estudio "tienen una condición móvil, la cual ha de redefinirse constantemente"10. La tesis según la cual la alta polarización de la sociedad chilena previa el golpe "llevó" a que se produjera el golpe de Estado de 1973 es rebatida en esta investigación, partiendo de la asunción que es parte del conocimiento científico ir "en contra de un conocimiento anterior, destruyendo conocimientos mal adquiridos" discutiendo y revisando eventos que en un período anterior fueron asumidos como verdades ${ }^{11}$.

A continuación, se lleva a cabo una revisión teórica de los conceptos de polarización y politización, se adopta una definición de los mismos y se propone una estrategia de medición empírica de dichos conceptos. Luego, se analizan los resultados empíricos obtenidos a partir de datos de encuestas de opinión de la época y se los pone en perspectiva con los hechos históricos acaecidos desde la llegada a la presidencia de Salvador Allende hasta su derrocamiento con el Golpe de 1973, para finalmente presentar las conclusiones.

\section{¿Qué es la polarización? ¿Cómo medirla?}

Existen ciertos países en los que, debido a la manera en que está conformado su sistema de partidos, el estudio y medición aplicada de la polarización está bastante avanzada, como pasa por ejemplo con Estados Unidos ${ }^{12}$. Por otro lado, se pueden

7. En todo caso la gran cantidad de encuestados que respondían en la encuesta Hamuy \#38 y \#45 (cerca del 80\%) al revisar periódicos diariamente sólo están afirmando la presencia de un alto grado de politización, pues son capaces de identificar los tópicos en disputas y los valores ideológicos en torno a los cuales se estructuran. 8 Bases de datos pertenecientes a ROPER, disponibles en https://ropercenter.cornell.edu/

9. Para más información sobre este tema de manera comparada en América Latina y Europa ver Bermeo, Nancy."Ordinary People in Extraordinary Times: The Citizenry and the Breackdown of Democracy", Princeton, Princeton University Press, 2013.

10. Torres Dujisin, 2014, op. cit., 19.

11. Gastón Bachelard. "La Formación de Espíritu Científico, Contribución a un Psicoanálisis del Conocimiento Objetivo", Buenos Aires, Siglo XXI Editores, 2000.

12. Dentro de los trabajos más reconocido sobre polarización en Estados Unidos está Morris Fiorina, Samuel Abrams y Jeremy Pope. "Culture War? The Myth of Polarized America”, Nueva York, Pearson Longman, 2004. Para un aproximación desde la sociedad civil ver Delia Baldassarri y Andrew Gelman."Partisans without Constraint: Political Polarization and Trends in American Public Opinion", American Journal of Sociology,Vol.114, No.2, Chicago, 2008, 408-446 y para una aproximación desde las élites ver a Keith Poole."Las raíces de la polarización de la política moderna en los Estados Unidos", Revista de ciencia política, Vol.28, No.3, Santiago, 
encontrar investigaciones que "hacen viajar" el concepto de polarización, llevando a cabo estudios comparados donde se mide la polarización a nivel de la élite ${ }^{13}$; trabajos donde se vincula la polarización como variable independiente que potencia la identificación partidaria, concluyendo que la percepción de un contexto polarizado por parte de los votantes los impulsa a identificarse partidariamente de manera más fuerte (en este caso se basan las conclusiones centrándose en Estados Unidos a partir de una encuesta panel a largo plazo) ${ }^{14}$; trabajos donde se identifica la polarización también como variable independiente que afecta la participación ${ }^{15}$ donde se sostiene que un electorado polarizado será más probable que apoye a partidos extremistas en democracias más nuevas que en países donde la democracia ya está establecida ${ }^{16}$.

Los trabajos que miden la polarización en Chile son escasos y, cuando lo hacen, es aplicando la medición de dicho concepto a nivel de los políticos o partidos políticos ${ }^{17}$ y no a nivel de la ciudadanía. La primera aproximación al concepto de polarización en términos básicos es la distancia entre dos extremos medida sobre una dimensión determinada ${ }^{18}$. A su vez, la medición de la polarización puede llevarse a cabo a partir del autoposicionamiento individual en torno a un eje ideológico (izquierda-derecha; liberalconservador; demócrata-republicano); a partir de la toma de posición en cuestiones relativas a la política interna o externa del país ${ }^{19}$ o a partir de la simpatía partidaria, habiendo posicionado los partidos políticos en competición en alguno de los ejes ideológicos ${ }^{20}$.

La orientación ideológica y la identificación partidaria son fuertes predictores de la intención de voto y de la aprobación presidencial21. En contextos de sistemas de partido estables como el chileno, la ideología y la identificación partidaria se utilizan intercambiadamente para conocer la autoubicación del electorado ${ }^{22}$. Por ello, para medir

2008, 3-37. Para una aproximación conceptual general ver a Marc Hetherington. "Putting Polarization in Perspective", British Journal of Political Science, Vol.39, No.2, Cambridge, 2009, 413-448

13. Se puede revisar la polarización en perspectiva comparada en Lupu, 2015, op. cit., 333. Y también en Manuel Alcántara Sáez y Cristina Rivas. "Elites parlamentarias y polarización en América Latina", VII Congreso Español de Ciencia política y de la Administración Política para un Mundo el cambio, 2007.

14. Idem.

15. Lawrence Ezrow, Margit Tavits y Jonathan Homola. "Voter polarization, strenght of partisanship and support for extremist parties", Comparative political Studies,Vol. 47, No.11, 2013, 1558-1583.

16. Idem.

17. Alcántara y Rivas, 2007, op. cit. Giovanni Sartori. "Partidos y sistemas de partidos: marco para un análisis", Madrid, Alianza Editorial S.A., 1980 (La primera edición del texto es de 1976, pero se consultó la versión en español correspondiente al año 1980).

18. Alcántara y Rivas, 2007, op. cit. Christopher Hare y Keith Poole. "The Polarization of Contemporary American Politics", Polity, Vol.46, No.3, Chicago, 2014, 411-429. Sanz Cazorla, Alberto. "La segunda ola de crispación: Polarización en la VIII Legislatura”, en José Ramón Montero e Ignacio Lago (eds.), Elecciones generales 2008. Centro de Investigaciones Sociológicas, Madrid, 2009.

19.Alcántara y Rivas, 2007, op. cit. Julieta Suárez-Cao. “Estados Unidos: crisis económica, reelección presidencial y polarización política”, Revista de Ciencia Política, Vol.33, No.1, Santiago, 2013, 185-205 (pág.193)

20. Alcántara y Rivas, 2007, op. cit. Sanz Cazorla, 2014, op. cit. Hetherington, 2009, op. cit. Lupu, 2015, op. cit. Fiorina, 2004, op. cit. Hare, 2014, op. cit.

21. Peter Mair. "Left-Right Orientations", en Russell Dalton y Hans-Dieter Klingemann (eds.), The Oxford Handbook of Political Behavior. Oxford, Oxford University Press, 2007.Para el caso chileno ver a Patricio Navia y Rodrigo Osorio “Make the Economy Scream?' Economic, Ideological and Social Determinants of Support for Salvador Allende in Chile 1970-3", Journal of Latin American Studies,Vol.49, No.4, Cambridge, 2017, 1-27.

22. Para ver trabajos que abordan el tema de la institucionalización del sistema de partidos chileno y estrategias para ser abordado metodológicamente Patricio Navia y Rodrigo Osorio “Encuestas de opinión pública antes de 1973", Latin American Research Review, Vol.50, No.1, Pensilvania, 2015, 117-139. Coppedge, Michael. "The Evolution of Latin American Party Systems," in Scott Mainwaring and Arturo Valenzuela, (eds.), Politics, Society, and Democracy: Latin America, Westview, 1998, 171-206. Luna, Juan Pablo. "Partidos Políticos y Sociedad en Chile. Trayectoria Histórica y Mutaciones Recientes", en Arturo Fontaine, Cristián Larroulet, Jorge Navarrete, 
la polarización en términos de autoposicionamiento individual, se ha optado por tomar la identificación partidaria ordenando los partidos políticos en base al eje de clasificación izquierda/derecha.

Uno de los pioneros en explorar y describir el concepto de polarización fue Anthony Downs ${ }^{23}$, quien se aproximó a dicho fenómeno observándolo y midiéndolo a nivel de los partidos políticos. Desde esta perspectiva se entiende que los partidos actúan de acuerdo a la probabilidad de obtener mayor cantidad de votos y en función de los incentivos contextuales que, en casos de polarización política, se ubican en los extremos del espectro político. En suma, se puede afirmar que el concepto de polarización política -en su definición mínima- se conoce como el posicionamiento de los actores políticos en polos ideológicos extremos, entendidos en términos de izquierda-derecha o liberalconservador ${ }^{24}$. A continuación, se presenta de manera gráfica dicha idea (ver figura1):

Figura 1: Distribución del posicionamiento político a lo largo del eje izquierda-derecha en un contexto polarizado

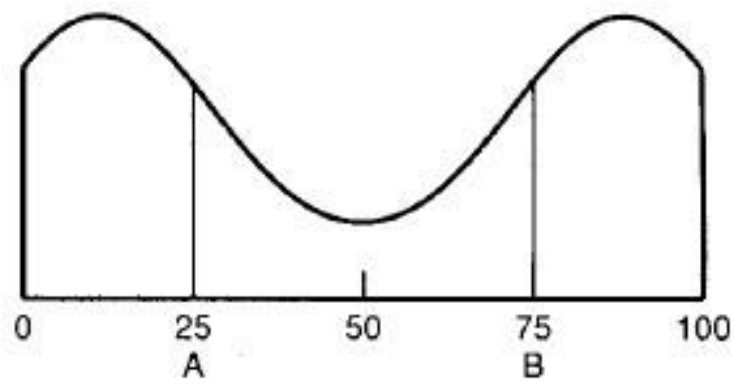

Fuente: Downs, 1957, op. cit., 143.

Es importante hacer hincapié en que todas las definiciones originarias de polarización, operacionalizaron dicho concepto a partir de su observación a nivel de las élites políticas, es decir, a partir de observar cómo se posicionaban los partidos políticos (y sus líderes) a lo largo de los ejes programáticos. De esta forma, por medio de la falacia ecológica, se tendió a inferir que la ciudadanía estaba polarizada a partir de observar, a nivel macro de los partidos políticos, dicha polarización. Esta estrategia se considera que tendió a sacar conclusiones poco acertadas respecto del posicionamiento ideológico del electorado. En esta investigación se rompe con la falacia ecológica al aterrizar la observación y medición de la polarización a nivel de los electores a partir de datos de encuestas.

Ignacio Walker (eds.), Reforma de los Partidos Políticos en Chile, Santiago, PNUD, 2008. Mainwaring, Scott y Scully, Timothy. "La construcción de las instituciones democráticas. Sistemas de partidos en América Latina", Santiago, CIEPLAN, 1995.

23. Anthony Downs. "Economic Theory of Political Action in a Democracy", Journal of Political Economy, Vol.65, No.2, Chicago, 1957, 135-150.

24. Hare, 2014, op. cit. Hetherington, 2009, op. cit. 


\section{Unidades de análisis y niveles de observación en el estudio de la polarización}

La observación tradicional de la polarización a nivel de los partidos políticos (dado el aglutinamiento en los polos ideológicos) significó interpretar que un cambio de partido en el gobierno produciría desajustes en la política del país. Según esta lógica, siempre habrá un partido político (situado al otro extremo del espectro ideológico) que se verá desfavorecido frente a la obtención de apoyos por parte de su contrincante; ya que "los partidos se alejarán hacia los extremos en vez de converger hacia el centro", pues desde el paradigma de la acción racional cada partido "obtiene más votos moviéndose hacia una posición radical de los que pierde en el centro"25.

Un contexto altamente polarizado sólo permitiría dos desenlaces posibles: el primero, un sistema con alternancia constante de partidos políticos que, dadas sus divisiones completamente opuestas, no conseguirían consolidar sus proyectos a largo plazo. El segundo escenario pronosticado es la superposición del grupo de oposición por sobre el oficialista, al no existir chances reales de llegar al poder. Desde esta perspectiva, en un sistema polarizado los partidos se mueven hacia los extremos más radicalizados porque tienen incentivos para hacerlo. Si se mantienen en el centro, la ganancia de votos es menor de la que obtienen radicalizando sus posiciones.

Sartori ${ }^{26}$ fue de los primeros en medir la polarización a nivel de la ciudadanía a partir de la identificación de un eje ideológico de izquierda-derecha y usando datos del Eurobarometer 1976. El clivaje izquierda-derecha es el que más tendencias acumula y que evidencia una relación directa en cómo ambos grupos conciben la política respecto a: justicia social y cómo llevar a cabo la política (status quo o no), simpatía por las potencias como Estados Unidos y la Unión Soviética, relevancia de la religiosidad en la política y simpatía respecto a grupos como sindicatos, clero, policía, empresas, movimientos sociales y grupos revolucionario ${ }^{27}$. Esto quiere decir que el eje izquierda-derecha abarca los temas fundamentales que implican la estructuración de la participación política en distintos países. Dicha estructuración programática en torno al autoposicionamiento izquierda-derecha coincide con los ejes sobre la base de los cuales se organizaba el electorado chileno para la época.

El autoposicionamiento en el eje suele medirse a partir de la opinión en torno a: 1) la intervención económica del Estado, entendiéndose que, a menor apoyo a la intervención estatal más a la derecha se posicionará el individuo; 2) el papel de la Iglesia, según el criterio por el cual mayores niveles de religiosidad coinciden con una visión más conservadora -y por tanto más de derecha-; y 3) las orientaciones ante el autoritarismo y la democracia, entendiendo que el apoyo al autoritarismo está vinculado a valores más conservadores $^{28}$. La dimensión económica, es decir, la mayor o menor intervención del Estado en la economía, parecería no mostrar un patrón estable respecto del posicionamiento izquierda-derecha en América Latina ${ }^{29}$. La intervención económica del Estado se refiere al papel del mismo como redistribuidor de la riqueza; mientras una

25. Ibid, 102.

26. Giovanni Sartori. "Polarización, fragmentación y competencia en las democracias occidentales", revista de Ciencia Política, Vol.13, No.1, Santiago, 1991, 39-73.

27. Ibid, 42-47.

28. Iván Llamazares y Rickard Sandell. "Partidos políticos y dimensiones ideológicas en Argentina, Chile, México y Uruguay. Esbozo de un análisis espacial”, Polis, No.99, México DF, 2003, 43-69.

29. Elizabeth Zechmeister y Margarita Corral. "El variado significado de izquierda y derecha en América Latina", Perspectivas desde el Barómetro de las Américas, No.38, Nashville, 2010 (pág.6) 
postura favorable a dicho rol redistribuidor del Estado es asociada a la izquierda (socialismo) la derecha se caracterizaría por una oposición a dicha intervención (neoliberalismo). En Chile quienes se identifican como de centro-izquierda son reacios a la intervención estatal de la economía ${ }^{30}$. En todo caso, para esa época de la historia política de Chile los ciudadanos latinoamericanos son capaces de autoubicarse en la escala izquierda-derecha en base a los criterios anteriormente expuestos ${ }^{31}$.

El grado de polarización en una sociedad, se mide a partir de la identificación de una fuerte presencia de ubicación en los extremos izquierda y derecha, además de un centro político vacío. En contextos actuales, Sanz Cazorla ya aplicó la medición de la polarización a nivel de la ciudadanía en España a partir del recuerdo de voto para el Partido Popular (PP) y el Partido Socialista Obrero Español (PSOE) ${ }^{32}$; Hetherington, para el caso de Estados Unidos, mide el grado de distancia ideológica entre los electores demócratas y republicanos a lo largo del tiempo ${ }^{33}$. Por tanto, existe acuerdo en que el criterio "distancia ideológica" entre partidos de izquierda y derecha es el más idóneo para observar empíricamente el grado de polarización ${ }^{34}$. No obstante Sartori considera que puede existir polarización en contextos donde existe una gran concentración en torno al centro ideológico introduciendo el concepto de pluralismo polarizado: "El sistema es multipolar en el sentido de que su mecánica competitiva depende de un centro que debe enfrentarse tanto con una izquierda como con una derecha"35.

Según Sartori, la idea de que un partido político de centro pueda estar presente en un sistema polarizado se sustenta en las dinámicas que estos desarrollan, pudiendo ejercer una fuerza centrípeta o centrífuga. Básicamente la fuerza centrípeta es la capacidad del sistema para atraer los partidos políticos hacia el centro y, a la inversa, la fuerza centrífuga es el desplazamiento de los partidos políticos desde el centro hacia los polos ideológicos ${ }^{36}$. Sartori señala el caso de Chile como uno de los ejemplos más claros de multipolaridad con una fuerza de centro centrífuga donde el Partido Democratacristiano (PDC o DC, partido emblemático de centro) habría sido incapaz de contener a los grupos más moderados dentro del centro político. Esta tesis aterrizada a nivel de los electores también rebatida aquí, al observarse la ausencia de posibles indicadores de fuerzas centrífugas tanto de quienes se dicen simpatizantes de la DC como de quienes se posicionaron en el centro en esa época.

En todo caso es coherente entender que la polarización sea evaluada a nivel de los electores en torno al grado de cercanía con los partidos políticos estructurados en torno al eje izquierda-derecha, y que su presencia se identifique mediante el distanciamiento ideológico de las fuerzas políticas extremas y a partir de la tendencia centrífuga entre los partidos posicionados al centro. Sartori, al aterrizar la medición de la polarización del nivel de las élites a las masas, entiende que el partido político es la representación institucional de los intereses de la sociedad, por tanto, se asume que en la evaluación dentro del eje izquierda-derecha debiese existir congruencia entre una élite y parte de la masa que lo apoya. La polarización puede ser evaluada de diversas maneras: como las

30. Llamazares y Sandell, 2003, op. cit., 48.

31. Idem. Zechmeister y Corral, 2010, op. cit. Manuel Alcántara. “¿Instituciones o máquinas ideológicas? Origen, programa y organización de los partidos políticos latinoamericanos", Barcelona, Instituto de Ciències Politiques i Socials, 2004. Para leer sobre autoposicionamiento político ver en Josep Colomer y Luis Escatel. "La dimensión izquierda-derecha en América Latina", Desarrollo Económico, Vol.22, No.177, Barcelona, 2005, 123-126.

32. Sanz Cazorla, 2014, op. cit.

33. Hetherington, 2009, op. cit.

34. Downs, 1957, op. cit. Sanz Cazorla, 2014, op. cit. Sartori, 1980, op. cit.

35. Sartori, 1980, op. cit.,171.

36. Ibid, 172. 
distancias y contigüidades percibidas por las élites políticas en términos de cuán cercanos, o a la inversa, cuán distintos sienten que son los otros partidos; o como la distancia resultante de un análisis de contenido de las plataformas electorales o posturas ideológicas de los partidos ${ }^{37}$.

Para precisar la coherencia entre el programa defendido por el partido y elector se asume que existirán desajustes menores, como la superposición parcial entre partidos dentro del eje y la similaridad ordinal de los partidos. La superposición de partidos se presenta cuando dos o más de ellos presentan valores afines en el eje izquierda-derecha, compartiendo un espacio (parcial) en común. Asimismo, la similaridad ordinal ocurre cuando las diferencias entre dos o más partidos recaen en otros tópicos, por lo que en el eje izquierda-derecha debieran estar en la misma posición ${ }^{38}$.

Pese a que se puede hablar de concordancia ideológica entre partidos y masas, no parece tan lógico poder afirmar que estén alienados en términos de la intensidad. Para el caso estadounidense, Fiorina argumenta que las masas parecen polarizadas porque los partidos políticos ofrecen opciones polarizadas ${ }^{39}$. Las preferencias de los votantes siguen siendo moderadas incluso en temas sociales controversiales. Por lo anterior, no existe acuerdo en que sea posible hablar de concordancia entre partidos políticos y masas para evaluar el caso de Chile previo al golpe de Estado de 1973. A nivel de élites es plausible pensar en la existencia de un sistema de partidos multipolar con un centro centrífugo, como estableció Sartori; sin embargo, a nivel de electores cabe preguntarse si éstos verdaderamente estaban siendo representados por los partidos políticos.

A partir de lo anterior, nace el interés del presente artículo, el cual pretende verificar si la polarización que se manifestaba a nivel de élites (partidos políticos) estaba reflejando la presencia de polarización a nivel del electorado. La pregunta que se desprende para el período de la historia de Chile entre 1970 y 1973 es: ¿ en qué medida la polarización ideológica manifestada a nivel la élite, ilustra el posicionamiento políticoideológico de las masas? es decir, para el periodo previo al golpe de Estado en Chile ¿estaban los ciudadanos polarizados ideológicamente como lo estaban las élites partidarias? Otros autores también se hacen esta pregunta y, al explorar el grado de aprobación a la gestión del Presidente Allende (en base a datos de la encuesta Hamuy) llegan a la conclusión negativa de dicha pregunta. En el presente trabajo se va más en profundidad, operacionalizando la medición de la polarización en términos clásicos, aterrizada a la ciudadanía y refutando la tesis de Sartori ${ }^{40} \mathrm{de}$ una multipolaridad centrífuga ${ }^{41}$.

\section{La estructura de la competencia partidaria previa al golpe: los tres tercios}

Para el período 1969-1973 en Chile, el número de los partidos políticos registrados en competencia oscilaba entre ocho y nueve ${ }^{42}$. Para ese mismo período el electorado era capaz de posicionarse en términos de simpatía y militancia respecto de los diferentes partidos en competición; así como a lo largo del eje izquierda-derecha ${ }^{43}$. Los partidos

37. Sartori, 1991, op. cit., 49.

38. Ibid, 54.

39. Fiorina, 2014, op. cit.

40. Sartori, 1980, op. cit.

41. Navia y Osorio, 2017, op. cit., 20.

42. Germán Urzúa. "Historia política de Chile y su evolución electoral (Desde 1810 a 1992)", Santiago, Editorial Jurídica de Chile, 1992 (pág.566)

43. Navia y Osorio, 2017, op. cit., 13. 
estaban altamente ideologizados y los programas políticos de los mismos eran claramente clasificables en la escala izquierda, centro y derecha; por ello en este trabajo, para alinear ideológicamente los partidos se tomó como referencia la medida en que los simpatizantes de los partidos se identificaban con la izquierda, la derecha o el centro ${ }^{44}$.

El sistema de partidos estaba permeado por el contexto del momento: "El que un sistema político tenga dos o más partidos depende de la distribución de votantes en la escala [izquierda-derecha]y de las normas electorales que rigen el sistema" 45 . En el Chile previo al golpe si bien existía un multipartidismo, dichos partidos políticos se organizaban en torno a la regla de los tres tercios; es decir que de forma más o menos equitativa los apoyos se repartían entre los líderes de la izquierda (Allende), el centro (Frei Montalva) y la derecha (Alessandri), cada uno de los líderes más representativos lograba en torno al 30\% de los votos hablándose así de una lógica de tres tercios ${ }^{46}$.

La estructuración de los apoyos políticos en tres tercios se debió por un lado a la pérdida de fuerza por parte de la derecha debido a sus "vacilaciones ideológicas" 47 , mientras que "la izquierda consiguió pasar de una situación de poder marginal a una situación de poder significativo" 48 . El centro abandona a sus representados y se alía con la derecha cuando "la cercanía programática (...) junto con la genuina búsqueda de una mayor democratización" situaban a la izquierda y al centro como posturas más en sintonía ${ }^{49}$. Desde la lógica de la polarización se entiende que, de forma progresiva, la izquierda fue acaparando apoyos, incrementando la oposición con la derecha y la trágica consecuencia de imponer una fuerza mayor para "restaurar" el equilibrio.

Pese al asumido consenso (tanto a nivel político como académico) respecto de la creciente distancia ideológica entre los simpatizantes de los partidos pertenecientes a ambos polos; existen pocos trabajos que midan empíricamente dicha distancia para cada período ${ }^{50}$. Para llevar a cabo el aterrizaje empírico de la observación de la polarización en una sociedad se puede hacer la medición a nivel empírico a partir del autoposicionamiento de los grupos en torno a un espectro ideológico dado que requiere la toma de posición previa respecto de ciertos tópicos que pueden ser polarizantes ${ }^{51}$; sin embargo la toma de posición previa sobre temas que pueden ser polarizantes forman parte de lo que aquí se entiende como politización. A continuación, se presentan los partidos políticos inscritos en la época y en torno a qué área del espectro político se alineaban (Tabla 1) para, de manera posterior, describir a nivel de electores los grados de simpatía que dichos partidos recibían.

44. Para ver otros trabajos que aplican una estrategia similar, Ibíd.

45. Downs, 1957, op. cit., 105.

46. Tomás Moulián e Isabel Torres, “¿Continuidad o cambio en la línea política del Partido Comunista de Chile?”, en Augusto Varas (compilador), El Partido Comunista de Chile. Estudio Multidisciplinario, Santiago, CESOCFLACSO, 1988 (pág.332).

47. Torres Dujisin, 2014, op. cit., 30.

48. Moulian y Torres Dujisin, 1989, op. cit., 23.

49. Torres Dujisin, 2014, op. cit., 26.

50. Ricardo Gamboa, Miguel Ángel López y Jaime Baeza "La evolución programática delos partidos chilenos 1970-2009: De la polarización al consenso", Revista de Ciencia Política, Vol. 33, No.2, Santiago, 2013, 443-467 (pág.444-445).

51. Iñaki Zagarzazu y Fermando Mouron. “Hugo Chavez’s polarizing legacy: chavismo, media, and public opinion in Argentina's domestic politics", Revista de ciencia política, Vol.37, No.1, Santiago, 2017, 147-175. 
Tabla 1: Partidos políticos en competición para las elecciones de 1970 en Chile

\begin{tabular}{|l|l|c|}
\hline \multicolumn{1}{|c|}{ IZQUIERDA } & \multicolumn{1}{|c|}{ CENTRO } & \multicolumn{1}{c|}{ DERECHA } \\
\hline Partido Comunista & Partido Demócrata Cristiano & Patria y Libertad \\
Partido Socialista & Partido Nacional \\
Partido Izquierda Radical & Democracia Radical \\
Movimiento de acción popular & & \\
Unitaria & & \\
Movimiento de Izquierda & & \\
Revolucionaria & & \\
Unión Socialista Popular & & \\
\hline
\end{tabular}

Fuente: Elaboración propia en base a los datos provistos por Urzúa, 1992, op. cit.

En términos de congruencia ideológica respecto de dónde se ubicaban los electores y en función de eso a quién apoyaban políticamente, Navia y Osorio ${ }^{52}$ describen (también a partir de resultados de la encuesta Hamuy), que los electores situados a la izquierda del espectro ideológico apoyaban a Allende; los situados en el centro (y adscritos al catolicismo) apoyaban a Tomic y los situados hacia la derecha conservadora (con mayor nivel educativo) apoyaban a Alessandri. Los porcentajes de apoyo a cada candidato están a su vez en congruencia con los niveles de apoyo electoral recibidos por cada candidato ${ }^{53}$.

\section{Resultados empíricos: la medición de la polarización y politización a nivel de electores en chile1970-1973}

Dado que el objetivo de investigación de este trabajo es conocer el grado de polarización política de la sociedad a partir de la identificación partidaria de las personas y el posicionamiento de cada partido político a lo largo del eje izquierda-derecha (Tabla 1), las fuentes de información más idóneas son las encuestas de opinión. En el inicio de la aplicación de encuestas electorales, la medición de la identificación partidaria sólo se realizaba consultando a los encuestados directamente respecto de la identificación con partidos políticos ${ }^{54}$. Para la misma época, pero en otros países, ciertos autores identifican el uso de encuestas de manera previa a la celebración de elecciones presidenciales; específicamente en Estados Unidos durante 1940 se consultaba a los votantes si eran republicanos o demócratas. En el sistema de representación chileno, en base a la estructuración de la competencia en los tres tercios, el cuestionario Hamuy preguntaba a quienes manifestaban no militar en ningún partido: "De los actuales partidos y Movimientos políticos ¿cuál es el que usted considera que se acerca más a sus ideas?" y, de forma posterior, preguntaba “ ¿Se siente usted más cerca de la derecha, de la izquierda o del centro?"55.

Previo a las elecciones de 1958 Hamuy llevó a cabo la primera encuesta preelectoral que arrojó un sondeo de las preferencias del electorado. Desde las elecciones presidenciales de 1964 realizó encuestas sistemáticas que acertaron de manera ajustada la victoria de Eduardo Frei Montalva. Para los siguientes comicios presidenciales -en el año 1970- Hamuy ejecutó la misma metodología, incluyendo preguntas relacionadas con

52. Navia y Osorio, 2017, op. cit.

53. Rodrigo Baño. "El nuevo carácter del apoliticismo", Serie de Estudios Políticos, No33, Santiago, FLACSO-Chile, 1995.

54. Budge, Ian; Crewe, Ivor; y Farlie, Dennis. "Party Identification and Beyond", Londres, ECPR Press, 1976.

55. Idem. 
el escenario internacional de la Guerra Fría. Sus resultados reflejaron el aumento de adhesión a la izquierda por parte de los chilenos, lo que se ratificó con el posterior triunfo de Salvador Allende. El cuestionario Hamuy no sólo preguntaba por identificación política, sino también por afinidad hacía líderes y partidos; así como respecto de acontecimientos que pudiesen dar indicios de posicionamiento político del encuestado.

A partir de los datos de la encuesta Hamuy, en este trabajo se medirá el concepto de "polarización a nivel del electorado" definida como "la disposición a ubicarse en los extremos del espectro político izquierda-derecha". El elemento "autoubicación" es clave y se distingue de la mera opinión respecto a temas de coyuntura política que se identifican con la politización. La politización es aquí definida como el proceso por el cual el individuo convierte una cuestión más o menos particular en un tema de interés público, ante el que asume que existen posiciones en conflicto ${ }^{56}$. Muñoz y Vilanova ${ }^{57}$ coinciden con este término como el acto de nombrar y considerar un asunto como político, agregando que la politización incluye "la controversia en torno a la aceptación de dicha acepción (definición del problema como público), y también el grado de apertura a la acción (interacción) de una arena política específica".

Es importante identificar en qué momento se está hablando de politización y en qué momento se empieza a hablar (y por tanto identificar) la polarización pues una requiere de la otra, es decir: para que pueda existir polarización (qué la ciudadanía se oponga, posicionándose discursivamente frente a un "otro") debe existir previamente politización (como la identificación de posiciones y temas en conflicto). No obstante, puede haber politización sin que ésta desemboque necesariamente en una polarización; por tanto, la politización es condición necesaria de la polarización. Puede existir politización sin polarización, mientras que la presencia de polarización delata la presencia previa de la politización. En base a la lógica de condición necesaria o suficiente ${ }^{58}$, la politización no es suficiente (aunque sí necesaria) para la presencia de polarización. De manera gráfica esto puede ser entendido mejor en la figura 2:

\section{Figura 2: La politización condición necesaria para que exista polarización}

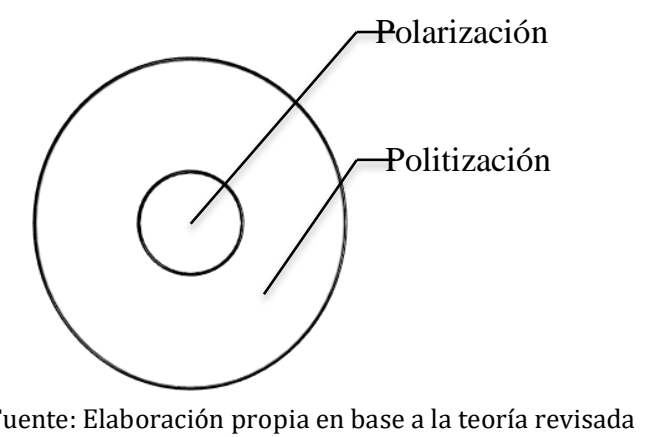

\footnotetext{
56. Jorge Benedicto y María Luz Morán.” ¿Otra clase de politización? Representaciones de la vida colectiva y procesos de implicación cívica de los jóvenes en situación de desventaja", Revista Internacional de Sociología, Vol.72, No.2, Córdoba, 2014, 429-452. También dentro de la sociología ver en María Jesús Funes "Procesos de socialización y participación comunitaria: estudio de un caso", Revista española de investigaciones sociológicas, No.67, Madrid, 1994, 187-206 (pág. 434)

57. Luz Muñoz y Pere Vilanova, "La politización de las organizaciones internacionales como proceso: una aproximación conceptual a sus determinantes y dinámicas", Revista Española de Ciencia Política, No.40, 2016, 139-160 (pág. 147).

58. Aníbal Pérez-Liñán. "El Método Comparativo y el análisis de configuraciones causales", Revista Latinoamericana de Política Comparada, Vol. No. 3, 2010, 125-148 (pág. 127)
} 
A la hora de medir empíricamente la polarización se hace evidente que dicho concepto necesita que quienes opinan se sientan ciudadanos, y por tanto implicados respecto de los procesos de coyuntura política con una postura formada al respecto (politizados) y, finalmente, se autoposicionen al respecto. Benedicto ${ }^{59}$, postula que en el proceso de politización deben estar presentes una serie de componentes: a) la aceptación de algún tipo de noción de "ciudadanía común"; b) la vinculación de los problemas individuales con ciertos sentimientos de justicia y de derechos; c) el reconocimiento, a través de la formulación de las demandas, como miembro de un grupo más amplio, en cuyo "nosotros común" estas mismas demandas ocupan un lugar destacado; d) la atribución de la responsabilidad y competencia a algún tipo de autoridad política, quien debe dar respuesta a las mismas; e) la expresión de las demandas a través de diferentes formas de acción colectiva, lo que les convierte a ellos mismos en actores colectivos.

Dado que la polarización se identifica a partir de la identificación de la necesaria politización previa, para poder ser medida se identifican dos dimensiones o aspectos: una que atañe al ámbito de la opinión (politización) y otra que atañe a la necesaria toma de posición al respecto (polarización). A continuación, se presenta en forma de tabla la manera en que se operacionalizan dichos conceptos para ser medidos a través de las preguntas que ofrece el cuestionario Hamuy (ver Tabla 2).

Tabla 2: Operacionalización conceptual de politización y polarización

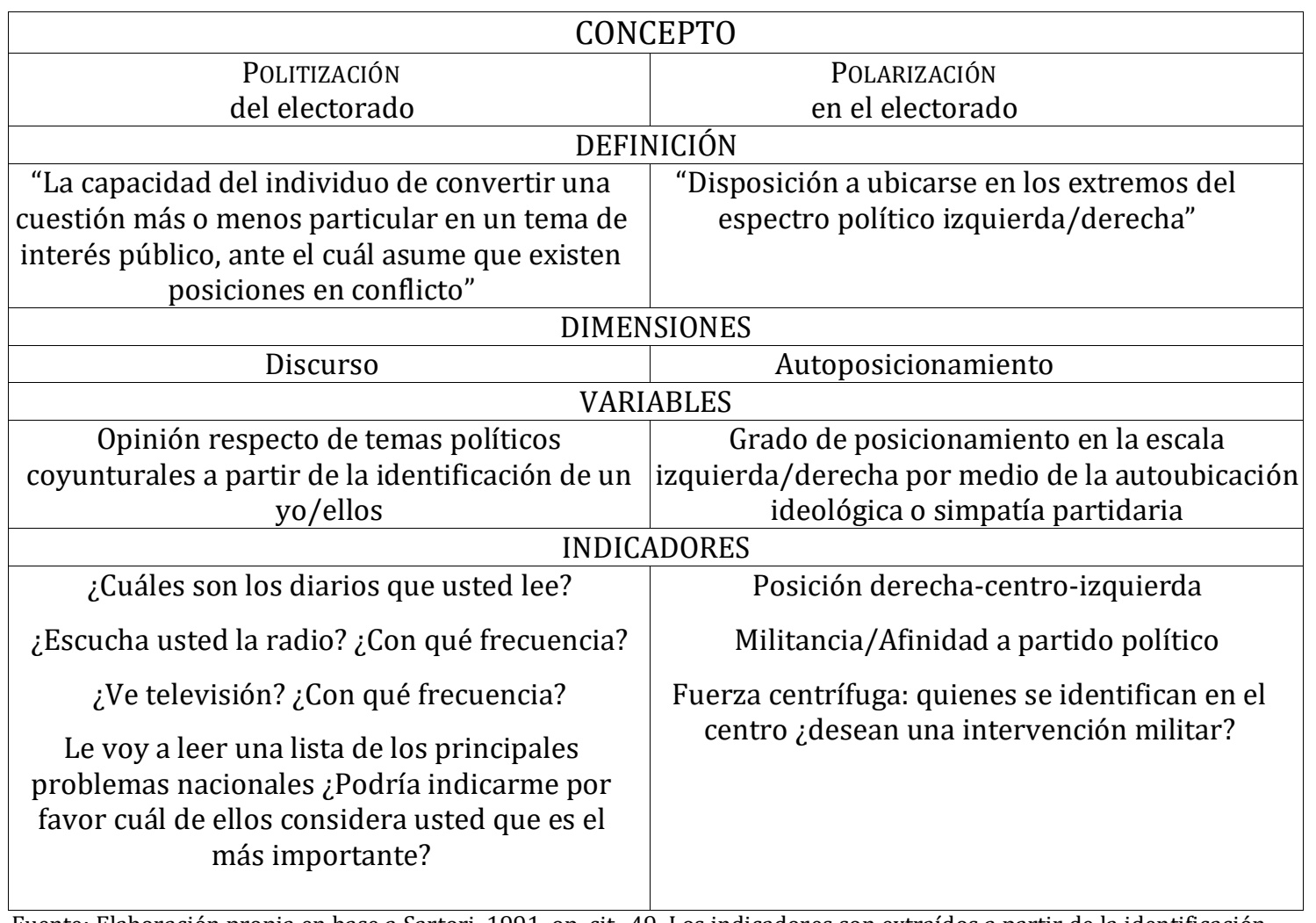

Fuente: Elaboración propia en base a Sartori, 1991, op. cit, 49. Los indicadores son extraídos a partir de la identificación de preguntas presentes en los cuestionarios de Hamuy \#38, \#42, \#43, \#44, \#45.

59. Benedicto, Jorge y Morán, María Luz. "¿Otra clase de politización? Representaciones de la vida colectiva y procesos de implicación cívica de los jóvenes en situación de desventaja", Revista Internacional de Sociología, Vol.72, No.2, Córdoba, 2014, 429-452 (pág. 7). 


\section{La sociedad chilena previa al golpe de 1973: muy politizada pero no polarizada}

La refutación de la asumida tesis de la polarización no es algo nuevo; Mattelart et al. ${ }^{60}$ dan evidencias ya en 1970 respecto de la presencia de una "ideología" de la clase dominante la cual se articulaba con la estrategia del enfrentamiento de clases. Preliminarmente, hay que especificar que aquí se entiende que la sociedad chilena comprendida entre los años 1970 y 1973 se encontraba altamente politizada, pero no existe evidencia que haya estado polarizada. Esto se puede corroborar a partir de los niveles de autoposicionamiento en el espectro ideológico que va de la izquierda a la derecha por parte de los encuestados. Tanto en los períodos cercanos a la celebración de las elecciones que llevaron a la presidencia a Salvador Allende, como en el período previo al Golpe de Estado en septiembre de 1973 existe una distribución equilibrada de porcentajes de apoyo a los partidos políticos tanto de izquierda, como de centro y derecha.

En el Gráfico 1 se pueden observar la identificación por partido político. La Democracia Cristiana es el partido que aglutina la mayor proporción de apoyos $(37,5 \%)$, siguiéndole en apoyo el Partido Socialista $(20,8 \%)$ y finalmente al Partido Nacional $(19,2 \%)$.

Gráfico 1: Identificación con los partidos políticos (porcentaje) agrupados en izquierda, centro y derecha.

Agosto 1970 (Hamuy \#38)

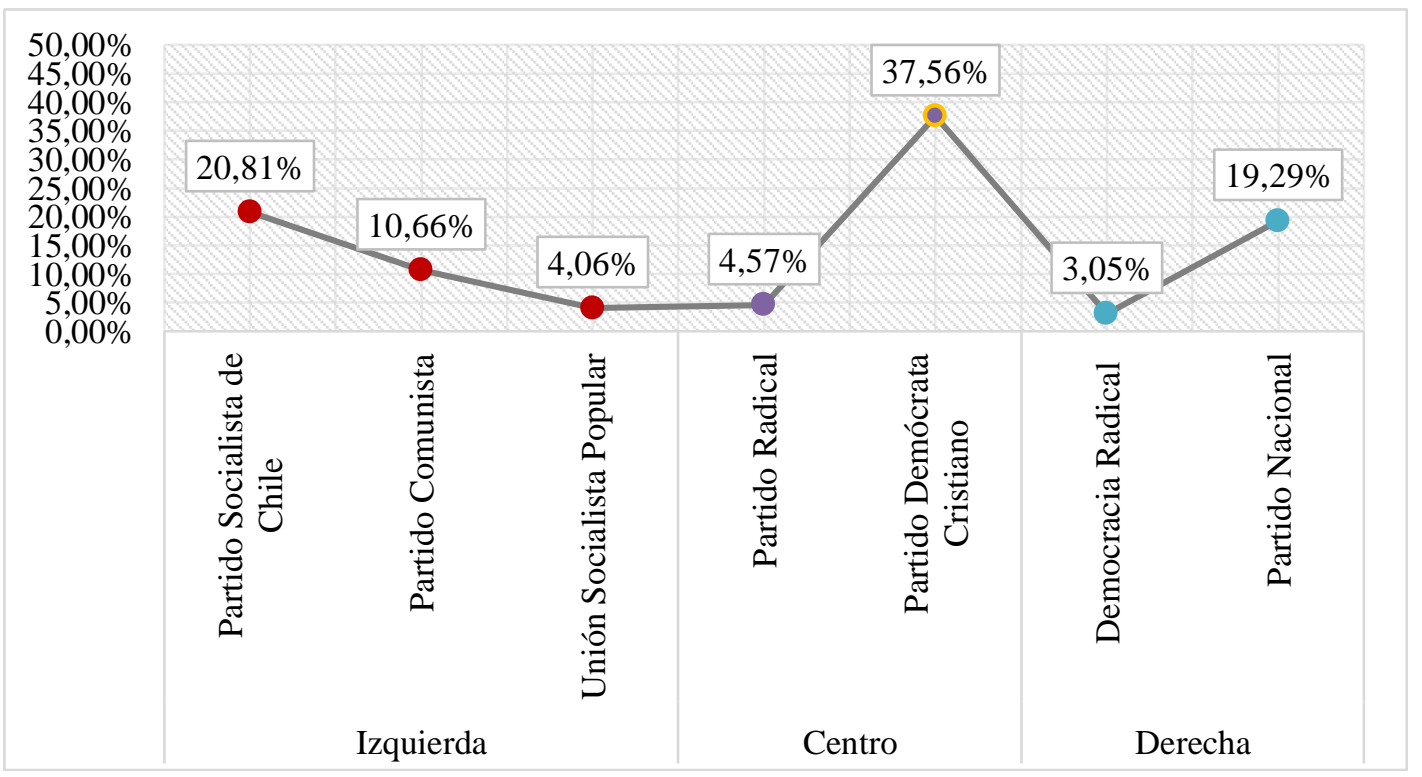

Fuente: Elaboración propia en base a los datos provistos por la encuesta Hamuy \#38 a partir de la pregunta "De los actuales partidos políticos, ¿cuál es el que usted considera que se acerca más a sus ideas?”. Respuestas omitidas en el gráfico (datos perdidos): "ninguno", "no sabe", "no responde" y "otros partidos".

Duverger ${ }^{61}$ postula que de un sistema bipartidista no surgen opiniones de centro que tenga una naturaleza distinta a la que proponen los otros dos partidos. Esto,

60. Armand Mattelart, Mabel Piccini y Michèle Mattelart. "Los medios de comunicación de masas. La ideología de la prensa liberal en Chile", Buenos Aires, El CID Editor, 1976 (pág.6).

61. Maurice Duverger. "Los partidos políticos", México, Fondo de Cultura Económica, 1957. 
extrapolado al caso chileno, y en un contexto aparentemente polarizado, quiere decir que, si había una izquierda y una derecha definidas una en oposición a la otra, cualquier partido naciente debiera provenir de uno de los dos bandos y, en consecuencia, no se debieran estar identificando (como ocurre a partir de los datos presentados) grandes concentraciones en torno a partidos de centro. De esta manera, la teoría en el caso de Chile se torna un poco más compleja, pudiendo interpretarse de dos maneras: 1) a partir de la identificación del Partido Democratacristiano (PDC o DC) como proveniente de un sector político que corresponde a los falangistas, es decir, de la derecha chilena; en función de dicho antecedente, el partido se posicionó en el centro del espectro político como tercera fuerza, pero siempre inclinado hacia la derecha dentro de la arena política. 2) Se puede interpretar que el PDC chileno tiene un carácter excepcional por cuanto efectivamente se aleja en el eje izquierda-derecha de sus raíces políticas, posicionándose en el centro del eje y refutando por tanto la hipótesis de una sociedad altamente polarizada

La segunda opción de interpretación es la que se adopta en esta investigación principalmente que: a) los encuestados que apoyan al PDC coinciden en definirse como de centro y b) porque desde la propia élite política de la DC, la derecha chilena en esa época no se estaba haciendo cargo del nuevo paradigma social de la época; a su vez la izquierda se estaba empapando de los pensamientos marxistas, alejados del catolicismo y sus valores. Es por ello que el Partido Democratacristiano se autodefine como una tercera vía, haciendo hincapié en que es completamente diferente a las fuerzas de izquierda y derecha ${ }^{62}$. Los niveles de auto ubicación con el centro ideológico por parte de la ciudadanía chilena se mantienen estables a lo largo del mandato del presidente Allende presentando un leve ascenso. En el gráfico 2 se puede observar dicha continuidad.

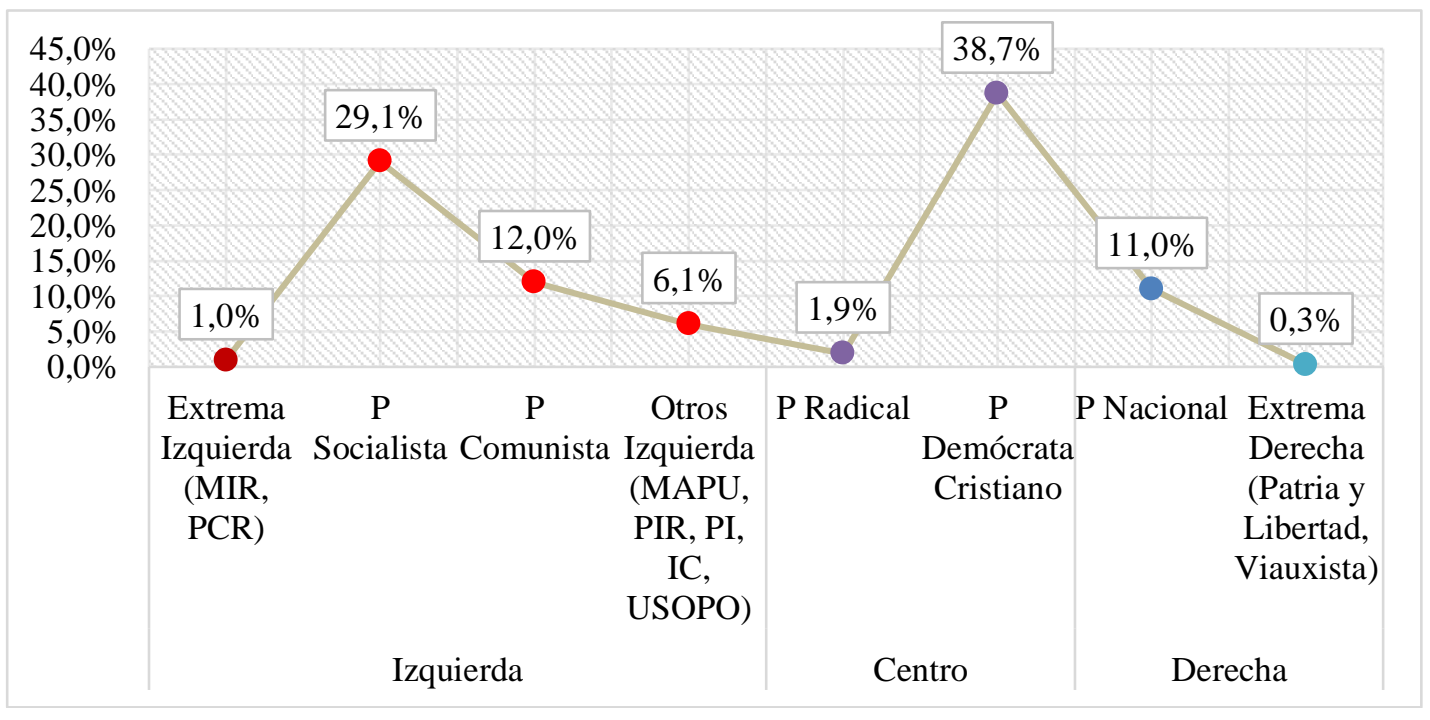

Fuente: Elaboración propia en base a los datos provistos por la encuesta Hamuy \#45 a partir de la pregunta "De los actuales partidos políticos, ¿cuál es el que usted considera que se acerca más a sus ideas?”. Respuestas omitidas en el gráfico (datos perdidos): "ninguno", "no sabe no responde", "otros partidos".

62. Michael Coppedge, 1998, op. cit. 
Los niveles de apoyo a los partidos políticos durante los meses previos al Golpe de Estado se aglutinan en torno a un $48 \%$ en la izquierda, $40,6 \%$ en el centro y $11,3 \%$ en la derecha. Navia y Osorio ${ }^{63}$ rescataron algunos de los resultados de la encuesta Hamuy, mediante análisis histórico y gráficos comparativos entre la predicción de Hamuy y resultados posteriores llegaron a dos aseveraciones relevantes que pueden guardar relación con la suposición de polarización política entre 1960 y 1973. En primer lugar, postularon que después del año 1964 aumentó la identificación con la izquierda. Si se testean las encuestas revisadas en su obra, efectivamente incrementa el porcentaje de personas que adhieren a este sector político. Sin embargo - y siguiendo la estricta definición de polarización- estos resultados no evidencian un contexto polarizado, ello porque el centro político sigue existiendo como una fuerza relevante en la arena política, no sólo como una ideología, sino que representado por dos partidos políticos: Partido Radical (PR) y Partido Democratacristiano (PDC). Es más, según las encuestas Hamuy\#36, Hamuy\#37 y Hamuy\#38, ante la pregunta de "identificación o afinidad con algún partido" es precisamente el PDC el partido que más representa a los encuestados.

En segundo lugar, se cuestiona la rigidez del centro político ${ }^{64}$. En efecto, si bien en años anteriores el Partido Radical había tenido el dominio de este espacio, ya a fines de la década de 1950 e inicios de 1960 el incipiente Partido Democratacristiano -de la mano de Eduardo Frei Montalva- adoptó un rol preponderante en el centro. "La DC se constituyó en una fuerza reformista capaz de 'contener el avance del comunismo' a través de una política interclasista, llegando a sectores sociales que creían que el único modo de conjurar la amenaza izquierdista era realizando cambios y favoreciendo una mayor equidad social"65. De esta manera el PDC abarcó el electorado que iba desde las clases más acomodadas, hasta pobladores de los campamentos "callampa" que surgieron a raíz de la migración campo- ciudad. Sin embargo, el hecho de que el centro político, al ser heterogéneo, pueda proveer votos tanto a la izquierda como a la derecha, no es una condición suficiente para sostener que la sociedad estuviera polarizada. En este sentido, si bien existieron posturas irreconciliables por la Guerra Fría, la idea democratacristiana de una "vía alternativa" enfocada en la asistencia social que no involucraba al marxismo, significó un punto intermedio entre posiciones opuestas. La existencia de un partido como el PDC supone que ciudadanos con pensamientos tan diferentes pudieran unirse en torno a una idea partidaria común.

La polarización entonces, corresponde a un fenómeno político que sólo se manifiesta en contextos politizados. Como ha sido estudiada la polarización es un fenómeno que usualmente se identificó a nivel de partidos políticos y sus dinámicas frente a determinados escenarios ${ }^{66}$. Sin embargo -tal como se ha manifestado aquí- no existe evidencia de que la lógica de polarización partidaria se pueda extrapolar a la ciudadanía. Para poder medir esta última, se evaluó mediante datos recopilados a través de encuestas que captaron el clima a este nivel vivido. La encuesta Hamuy realizada en el periodo previo a 1973, otorgó la información adicional, midiendo posturas e impresiones concretas de la ciudadanía más allá de la toma de decisiones adoptadas por la clase política.

63. Navia y Osorio, 2015, op. cit.

64. Idem.

65. Torres-Dujisin, 2014, op. cit., 141.

66. Downs, 1957, op. cit. Duverger, 1957, op. cit. Sartori, 1991, op. cit. 
La tesis de Sartori ${ }^{67}$ respecto del caso chileno, el cual definió como un sistema multipolar con un centro centrífugo, se ve refutada al verificar que los encuestados que apoyaban al PDC (situados en el centro del espectro político) están en contra (en torno a un 70\% de manera constante) de la conveniencia de un Gobierno Militar.

Gráfico 3: Niveles de acuerdo respecto de la conveniencia de un Gobierno Militar entre los encuestados posicionados en el centro del espectro ideológico.

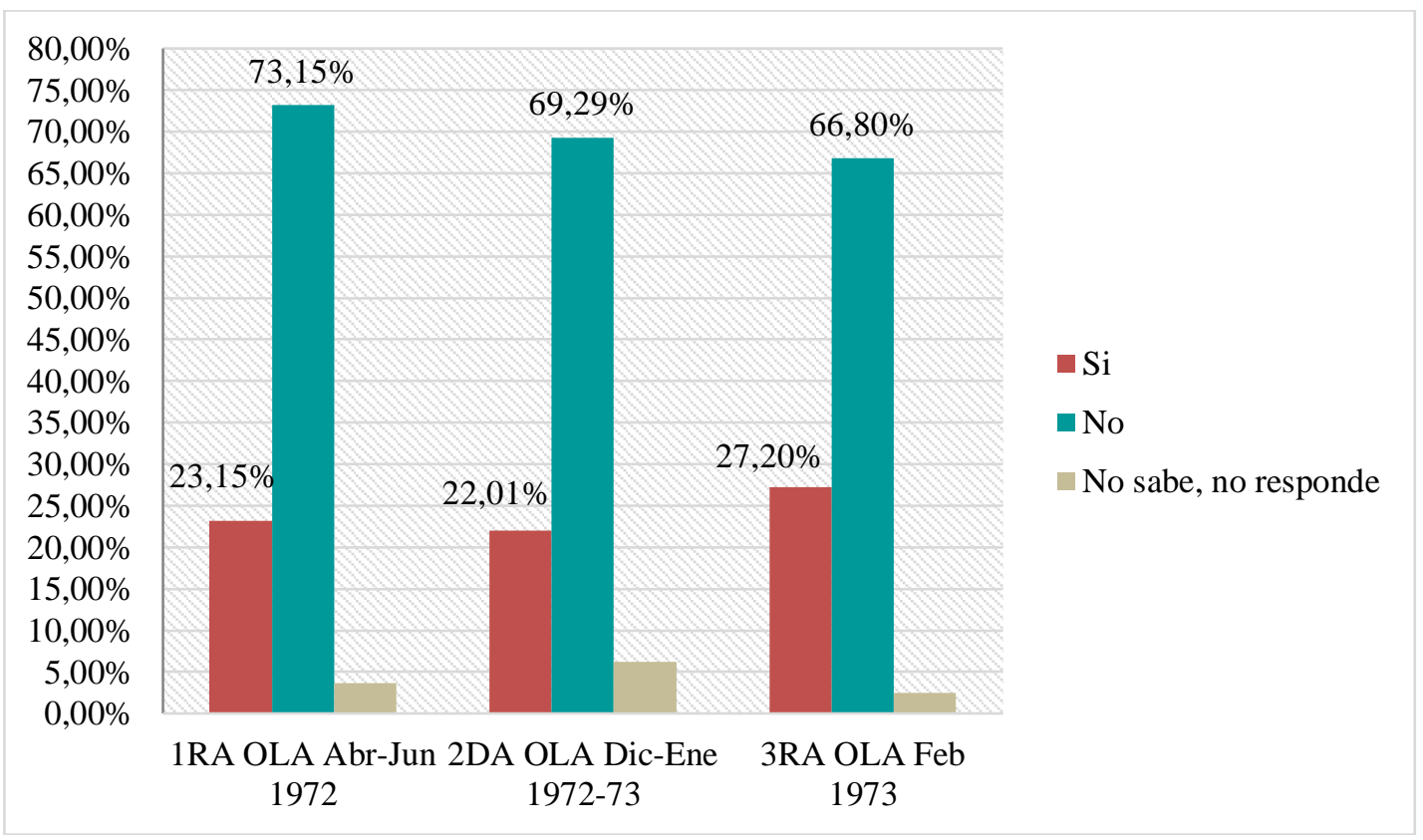

Fuente: Elaboración propia en base a la pregunta "¿Cree usted que un gobierno militar es conveniente para Chile?". Porcentaje de respuesta sólo seleccionados los encuestados que se posicionaron al centro (previo al golpe) Hamuy \#43, \#44, \#45.

\section{Conclusiones}

A partir de los resultados presentados se concluye que, si bien existía una alta politización a nivel de la ciudadanía durante el período previo al Golpe de Estado en Chile, dicha politización no se tradujo necesariamente en polarización política, entendida como la presencia de dos fuerzas contrapuestas en términos ideológicos a partir de la autoubicación en los extremos del espectro izquierda-derecha (o liberal-conservador). A su vez, se refuta la presencia de un centro ideológico de carácter centrífugo como lo postuló Sartori ${ }^{68}$, ya que a nivel de encuestados posicionados en el centro ideológico, se constata un rechazo alto y estable ante una eventual intervención militar (en torno al 70\% constantes a lo largo de las encuestas revisadas).

No obstante, la polarización política a nivel de la élite es innegable y es a este nivel desde cual se articuló y llevó a cabo el desenlace final del Golpe de Estado al Gobierno de Allende en 1973. Al examinar el fenómeno de la polarización a distintos niveles de observación, queda en evidencia la importancia de hacer dialogar las investigaciones que se aproximan al mismo objeto de estudio. Si bien la interrupción de la democracia para

67. Sartori, 1980, op. cit. 68. Idem. 
instaurar una dictadura nunca puede ser justificado; en base a los supuestos normalmente asumidos: si la "necesidad" de un Golpe de Estado no estaba fundada en una polarización política de la sociedad ¿por qué se llevó a cabo dicho quiebre democrático? Ésta y otras preguntas se abren luego de presentar estos resultados: sería interesante seguir indagando si quienes participaron en primera persona durante esos años ¿aumentaron su militancia en los partidos políticos? ¿aumentaron la participación en movilizaciones de peticiones y protestas?

Más allá de las preguntas abiertas, se considera que esta investigación está haciendo un aporte a la investigación en el proceso de respuesta a las preguntas antes planteadas al aportar fundamentación empírica. Por lo anterior, se entiende que el presente trabajo viene a complementar el estudio de la polarización desde los partidos políticos; entendiendo que el objetivo del conocimiento científico es ir "en contra de un conocimiento anterior, destruyendo conocimientos mal adquiridos" a partir de complementar la discusión en torno a eventos asumidos como verdades ${ }^{69}$.

\section{Bibliografía}

Alcántara, Manuel. “¿Instituciones o máquinas ideológicas? Origen, programa y organización de los partidos políticos latinoamericanos", Barcelona, Instituto de Ciències Politiques i Socials, 2004.

Alcántara, Manuel y Rivas, Cristina. "Elites parlamentarias y polarización en América Latina", VII Congreso Español de Ciencia política y de la Administración Política para un Mundo el cambio, 2007, disponible en http://www.aecpa.es/uploads/files/congresos/congreso 08/area3/GT-8/ALCANTARAMANUEL.pdf

Bachelard, Gastón. "La Formación de Espíritu Científico, Contribución a un Psicoanálisis del Conocimiento Objetivo", Buenos Aires, Siglo XXI Editores, 2000.

Baldassarri, Delia y Gelman, Andrew. "Partisans without Constraint: Political Polarization and Trends in American Public Opinion", American Journal of Sociology, Vol.114, No.2, Chicago, 2008, 408-446.

Baño, Rodrigo. "El nuevo carácter del apoliticismo", Serie de Estudios Políticos, No33, Santiago, FLACSO-Chile, 1995.

Benedicto, Jorge; Fernández de Mosteyrín, Laura; Funes, María Jesús; Monferrer, Jordi; Morán, María Luz; Robles, José Manuel. "La construcción del sujeto político en la juventud: El caso de los jóvenes andaluces en riesgo", Actualidad, Vol. 58, Sevilla, 2011, 1-29

Benedicto, Jorge y Morán, María Luz. "¿Otra clase de politización? Representaciones de la vida colectiva y procesos de implicación cívica de los jóvenes en situación de desventaja", Revista Internacional de Sociología, Vol.72, No.2, Córdoba, 2014, 429-452.

Bermeo, Nancy. "Ordinary People in Extraordinary Times: The Citizenry and the

69. Bachelard, 2000, op. cit. 
Breackdown of Democracy", Princeton, Princeton University Press, 2013.

Bernedo, Patricio y Porath, William. "A tres décadas del golpe: ¿Cómo contribuyó la prensa al quiebre de la democracia chilena?" Cuadernos de Información. No.16-17, Santiago, 20032004, 114-124.

Budge, Ian; Crewe, Ivor; y Farlie, Dennis. "Party Identification and Beyond", Londres, ECPR Press, 1976.

Colomer, Josep y Escatel, Luis. "La dimensión izquierda-derecha en América Latina", Desarrollo Económico, Vol.22, No.177, Barcelona, 2005, 123-126.

Coppedge, Michael. "The Evolution of Latin American Party Systems," in Scott Mainwaring and Arturo Valenzuela, (eds.), Politics, Society, and Democracy: Latin America, Westview, $1998,171-206$.

Downs, Anthony. "Economic Theory of Political Action in a Democracy", Journal of Political Economy, Vol.65, No.2, Chicago, 1957, 135-150.

Duverger, Maurice. "Los partidos políticos", México, Fondo de Cultura Económica, 1957.

Ezrow, Lawrence; Tavits, Margit y Homola, Jonathan. "Voter polarization, strenght of partisanship and support for extremist parties", Comparative political Studies,Vol. 47, No.11, 2013,1558-1583.

Faure, Antoine. "Par temp sagités Temporalités journalistiques et crise politiqueau Chili (1970-1990)", Temporalite, 2016, Vol.23, 1-26

Fiorina, Morris; Abrams, Samuel y Pope, Jeremy. "Culture War? The Myth of Polarized America", Nueva York, Pearson Longman, 2004

Funes, María Jesús. "Procesos de socialización y participación comunitaria: estudio de un caso”, Revista española de investigaciones sociológicas, No.67, Madrid, 1994, 187-206.

Gamboa, Ricardo; López, Miguel Ángel y Baeza, Jaime. "La evolución programática delos partidos chilenos 1970-2009: De la polarización al consenso", Revista de Ciencia Política, Vol. 33, No.2, Santiago, 2013, 443-467.

Gronemeyer, María Elena yPorath, William. "Tendencias de la posición editorial en diarios de referencia en Chile. El arte de dosificar la crítica frente a la actuación de los actores políticos", Revista de Ciencia Política,Vol. 37, No.1, Santiago, 2017, 177-202.

Hare, Christopher y Poole, Keith. "The Polarization of Contemporary American Politics", Polity,Vol.46, No.3, Chicago, 2014, 411-429.

Hetherington, Marc. "Putting Polarization in Perspective", British Journal of Political Science, Vol.39, No.2, Cambridge, 2009, 413-448.

Linz, Juan. "The Breakdown of Democratic Regimes: Crisis, Breakdown 
AndReequilibration", Baltimore, Johns Hopkins University Press, 1978.

Llamazares, Iván y Sandell, Rickard. "Partidos políticos y dimensiones ideológicas en Argentina, Chile, México y Uruguay. Esbozo de un análisis espacial", Polis, No.99, México DF, 2003, 43-69.

Luna, Juan Pablo. "Partidos Políticos y Sociedad en Chile. Trayectoria Histórica y Mutaciones Recientes", en Arturo Fontaine, Cristián Larroulet, Jorge Navarrete, Ignacio Walker (eds.), Reforma de los Partidos Políticos en Chile, Santiago, PNUD, 2008.

Lupu, Noam. "Party Polarization and Mass Partisanship: A Comparative Perspective", Polital Behavior, Vol.37, No.2, Kentucky, 2015, 331-356.

Mainwaring, Scott y Scully, Timothy. "La construcción de las instituciones democráticas. Sistemas de partidos en América Latina", Santiago, CIEPLAN, 1995.

Mair Peter. "Left-Right Orientations", en Russell Dalton y Hans-Dieter Klingemann (eds.), The Oxford Handbook of Political Behavior. Oxford, Oxford University Press, 2007.

Mattelart, Armand; Piccini, Mabel y Mattelart, Michèle."Los medios de comunicación de masas. La ideología de la prensa liberal en Chile", Buenos Aires, El CID Editor, 1976.

Moulián, Tomás y Torres, Isabel. “¿Continuidad o cambio en la línea política del Partido Comunista de Chile?", en Augusto Varas (compilador), El Partido Comunista de Chile. Estudio Multidisciplinario, Santiago, CESOC-FLACSO, 1988.

Muñoz, Luz y Vilanova, Pere. "La politización de las organizaciones internacionales como proceso: una aproximación conceptual a sus determinantes y dinámicas", Revista Española de Ciencia Política, No.40, 2016, 139-160.

Navia, Patricio y Osorio, Rodrigo. "Encuestas de opinión pública antes de 1973", Latin American Research Review, Vol.50, No.1, Pensilvania, 2015, 117-139.

Navia, Patricio y Osorio, Rodrigo. “Make the Economy Scream?' Economic, Ideological and Social Determinants of Support for Salvador Allende in Chile 1970-3", Journal of Latin American Studies, Vol.49, No.4, Cambridge, 2017, 1-27.

Pérez-Liñán, Aníbal. "El Método Comparativo y el análisis de configuraciones causales", Revista Latinoamericana de Política Comparada, Vol. 3, 2010, 125-148

Poole, Keith. "Las raíces de la polarización de la política moderna en los Estados Unidos", Revista de ciencia política, Vol.28, No.3, Santiago, 2008, 3-37.

Sanz Cazorla, Alberto. "La segunda ola de crispación: Polarización en la VIII Legislatura", en José Ramón Montero e Ignacio Lago (eds.), Elecciones generales 2008. Centro de Investigaciones Sociológicas, Madrid, 2009.

Sartori, Giovanni. "Partidos y sistemas de partidos: marco para un análisis”, Madrid, Alianza Editorial S.A., 1980. 
Sartori, Giovanni. "Polarización, fragmentación y competencia en las democracias occidentales", Revista de Ciencia Política, Vol.13, No.1, Santiago, 1991, 39-73.

Suárez-Cao, Julieta. "Estados Unidos: crisis económica, reelección presidencial y polarización política", Revista de Ciencia Política, Vol.33, No.1, Santiago, 2013, 185-205.

Torres, Isabel. "La Crisis del Sistema Democrático: las elecciones presidenciales y los proyectos políticos excluyentes. Chile 1958-1970", Santiago, Editorial Universitaria, 2014.

Urzúa, Germán. "Historia política de Chile y su evolución electoral (Desde 1810 a 1992)”, Santiago, Editorial Jurídica de Chile, 1992.

Valenzuela, Arturo. "Democracy, Politics, and Society Latin America: Essays in Honor of Juan Linz", Boulder, Westview Press, 2003.

Zagarzazu, Iñaki y Mouron, Fernando. "Hugo Chavez's polarizing legacy: chavismo, media, and public opinion in Argentina's domestic politics", Revista de ciencia política, Vol.37, No.1, Santiago, 2017, 147-175.

Zechmeister, Elizabeth y Corral, Margarita. "El variado significado de izquierda y derecha en América Latina", Perspectivas desde el Barómetro de las Américas, No.38, Nashville, 2010.

\section{OTROS RECURSOS}

Base de Datos Encuestas Hamuy, ROPER. Rescatado de https://ropercenter.cornell.edu/ 